\title{
Serum neutrophil gelatinase-associated lipocalin at 3 hours after return of spontaneous circulation in patients with cardiac arrest and therapeutic hypothermia: early predictor of acute kidney injury
}

Yoon Hee Choi ${ }^{1}$, Dong Hoon Lee ${ }^{2}$ and Jae Hee Lee ${ }^{1 *}$ (D)

\begin{abstract}
Background: Serum neutrophil gelatinase-associated lipocalin (NGAL) could be used as a predictive marker of acute kidney injury (AKI) in patients with return of spontaneous circulation (ROSC) after out-of-hospital cardiac arrest (OHCA) who are managed with targeted temperature management (TTM). However, the NGAL measurement timepoints vary from immediately after ROSC to several days later. The primary objective of this study was to determine an association between AKI and NGAL, both immediately (ROSC-NGAL) and $3 \mathrm{~h}$ after ROSC ( $3 \mathrm{~h}-\mathrm{NGAL}$ ), in OHCA patients with TTM. The secondary objective was to ascertain the association between NGAL levels in the early post-ROSC phase and the neurologic outcomes at discharge.
\end{abstract}

Methods: This prospective observational study was conducted between January 2016 and December 2018 and enrolled adult OHCA patients ( $\geq 18$ years) with TTM after ROSC. The serum NGAL level was measured both immediately and $3 \mathrm{~h}$ after ROSC. Univariate and multivariate analyses were performed to identify the associations between AKI, poor neurologic outcome, and NGAL.

Results: Among 861 OHCA patients, 89 patients were enrolled. AKI occurred in 48 (55.1\%) patients. On multivariate logistic regression analysis, 3 h-NGAL was significantly associated with AKI (odds ratio [OR] 1.022; 95\% confidence interval [CI] 1.009-1.035; $p=0.001)$. The area under the receiver operating characteristic curve of $3 \mathrm{~h}-\mathrm{NGAL}$ for AKI was $0.910(95 \%$ Cl 0.830-0.960), and a cut-off value of $178 \mathrm{ng} / \mathrm{mL}$ was identified. Both ROSC-NGAL and $3 \mathrm{~h}-\mathrm{NGAL}$ were not significantly associated with poor neurologic outcome on multivariate logistic regression analysis (ROSC-NGAL; OR 1.017; 95\% Cl 0.998-1.036; $p=0.084$, 3 h-NGAL; OR 0.997; 95\% Cl 0.992-1.001; $p=0.113$ ).

\footnotetext{
* Correspondence: jaeheelee.md@hanmail.net

'Department of Emergency Medicine, College of Medicine, Ewha Womans University Mokdong Hospital, 1071 Anyangcheon-ro, Yangcheon-gu, Seoul 07985, South Korea

Full list of author information is available at the end of the article
}

(C) The Author(s). 2020 Open Access This article is licensed under a Creative Commons Attribution 4.0 International License, which permits use, sharing, adaptation, distribution and reproduction in any medium or format, as long as you give appropriate credit to the original author(s) and the source, provide a link to the Creative Commons licence, and indicate if changes were made. The images or other third party material in this article are included in the article's Creative Commons licence, unless indicated otherwise in a credit line to the material. If material is not included in the article's Creative Commons licence and your intended use is not permitted by statutory regulation or exceeds the permitted use, you will need to obtain permission directly from the copyright holder. To view a copy of this licence, visit http://creativecommons.org/licenses/by/4.0/ The Creative Commons Public Domain Dedication waiver (http://creativecommons.org/publicdomain/zero/1.0/) applies to the data made available in this article, unless otherwise stated in a credit line to the data. 
(Continued from previous page)

Conclusions: The serum NGAL concentration measured $3 \mathrm{~h}$ after ROSC is an excellent early predictive marker for AKI in OHCA patients treated with TTM. Future research is needed to identify the optimal measurement timepoint to establish NGAL as a predictor of neurologic outcome and to validate the findings of this research.

Keywords: Acute kidney injury, Out-of-hospital cardiac arrest, Targeted temperature management, Serum neutrophil gelatinase-associated lipocalin

\section{Background}

The post-cardiac arrest syndrome (PCAS) develops in cardiac arrest patients after the return of spontaneous circulation (ROSC) and comprises anoxic brain injury, postcardiac arrest myocardial dysfunction, systemic ischemiareperfusion response, and persistent precipitating pathology [1]. The ischemia-reperfusion response of PCAS could injure various organs, including the kidneys, which in turn could lead to AKI [2]. AKI occurs in approximately half of patients with PCAS and is associated with poor clinical outcome [3-7]. The serum creatinine level is the gold standard diagnostic criterion for AKI. However, one of the limitations with the use of the serum creatinine level as a diagnostic criterion is its inability to indicate mild/early-stage renal injury [8]. Neutrophil gelatinase-associated lipocalin (NGAL), which is one of the most researched biologic markers of AKI, have clinical utility as an early marker of AKI [9]. The evidence from the previous research indicates that NGAL could facilitate an AKI diagnosis in adult critically ill patients $48 \mathrm{~h}$ earlier than the Risk, Injury, and Failure, and Loss, and End-stage kidney disease, or RIFLE, criteria [10]. NGAL was thought to be produced in the kidneys [11]; however, a recent study has reported different results. In a basic research study by Skrypnyk et al., interleukin-6 (IL-6) was shown to mediate hepatic NGAL production in AKI in a mice. Those authors reported that hepatocytes are the primary source of plasma and urine NGAL during AKI [12]. However, AKI is a highly complex systemic disorder, and there are limitations with regard to the generalizability of the abovementioned results.

Several reports of the positive association between AKI and NGAL in post-cardiac arrest patients indicate that NGAL could be used as a predictive marker of AKI; however, in those studies, the timepoint of NGAL measurement varied from immediately after ROSC to several days later [13-15]. If the NGAL level in early-stage of ROSC is correlated with the occurrence of AKI, then, this would enable the prediction of $\mathrm{AKI}$ and the early initiation of appropriate management.

This study aimed to evaluate the correlation between NGAL and AKI to evaluate the use of NGAL as a predictive marker for AKI in patients with PCAS. The primary objective of this study was to determine an association between AKI and NGAL, both immediately and $3 \mathrm{~h}$ after ROSC, in out-of-hospital cardiac arrest (OHCA) patients who underwent targeted temperature management (TTM) after ROSC. The secondary objective was to ascertain the association between NGAL levels in the early post-ROSC phase and the neurologic outcomes at discharge.

\section{Methods}

\section{Study setting and data collection}

This prospective observational study was conducted at single tertiary hospital in Seoul, South Korea between January 2016 and December 2018. The target study population comprised all adult OHCA patients (age $\geq 18$ years) who underwent TTM after ROSC. Patients with active intracranial bleeding, a do-not-resuscitate order, underlying disease with life expectancy $<6$ months, prearrest cerebral performance category of 3 or 4 , body temperature $<30^{\circ}$ (the abovementioned contraindications for TTM [16]), end-stage renal disease, and missing data on NGAL measurements were excluded.

All study participants underwent post-cardiac arrest care and TTM in accordance with standardised institutional protocol. Baseline patient information and clinical data were collected through a chart review of the electronic medical records, whereas the data on the 1 - and 6-month post-discharge survival were obtained by telephonic follow-up and accordingly recorded. If the patient died during the follow-up period, the date of death was recorded.

This study was approved by the institutional review board of Ewha Womans University Mokdong Hospital.

\section{Outcome measures}

The primary outcome was the occurrence of AKI during hospitalisation. AKI was diagnosed on the basis of the Kidney Disease Improving Global Outcomes (KDIGO) guidelines, by using the serum creatinine level and urinary output [17]. Thus, AKI was defined based on any of the following: increase in serum creatinine ( $\mathrm{SCr}$ ) by $\geq 0.3$ $\mathrm{mg} / \mathrm{dL}(26.5 \mu \mathrm{mol} / \mathrm{L})$ within $48 \mathrm{~h}$; increase in $\mathrm{SCr}$ to $\geq 1.5$ times the baseline, which is known or presumed to have occurred within the past 7 days; or urinary volume $<0.5$ $\mathrm{mL} / \mathrm{kg} / \mathrm{h}$ for $6 \mathrm{~h}$. The severity of AKI was determined according to the following criteria: Stage 1, increase in $\mathrm{SCr}$ up to 1.5 to 1.9 times the baseline value or increase in $\mathrm{SCr} \geq 0.3 \mathrm{mg} / \mathrm{dL}(\geq 26.5 \mu \mathrm{mol} / \mathrm{L})$ or urine output $<0.5$ $\mathrm{mL} / \mathrm{kg} / \mathrm{h}$ for $6-12 \mathrm{~h}$; Stage 2, increase in SCr 2.0 to 2.9 
times the baseline value or urine output $<0.5 \mathrm{~mL} / \mathrm{kg} / \mathrm{h}$ for $\geq 12 \mathrm{~h}$; Stage 3, increase in SCr to 3.0 times the baseline value or $\mathrm{SCr} \geq 4.0 \mathrm{mg} / \mathrm{dL}(\geq 353.6 \mu \mathrm{mol} / \mathrm{L})$ or initiation of renal replacement therapy or decreased eGFR to $<35 \mathrm{~mL} /$ $\mathrm{min} / 1.73 \mathrm{~m}^{2}$ in patients younger than 18 years or with urine output $<0.3 \mathrm{~mL} / \mathrm{kg} / \mathrm{h}$ for $\geq 24 \mathrm{~h}$ or anuria for $\geq 12 \mathrm{~h}$. For patients who were treated at the study centre before the cardiac arrest event or with information available on the creatinine level through medical records from another hospital, the previously recorded creatinine level was used as the baseline value. In patients without a previous creatinine level, the lowest value from tests performed within $24 \mathrm{~h}$ after ROSC was used as the baseline value. Creatinine was measured immediately after ROSC and again at $3 \mathrm{~h}$ after ROSC. After being admitted to the intensive care unit, laboratory tests were conducted daily or two times a day. The secondary outcome was the neurologic outcome at discharge, which was measured by using the Cerebral Performance Category (CPC) score that comprises five categories: good recovery (CPC 1), moderated disability (CPC 2), severe disability (CPC 3 ), vegetative state (CPC 4), and brain death or death (CPC 5) [18]. In this study, good neurologic outcome (GNO) was categorised as CPC 1 and 2 and poor neurologic outcome (PNO) as CPC 3-5.

The NGAL, which is an acute-phase protein after ischemic or nephrotoxic AKI, can be measured in urine or serum samples [19], and the serum NGAL can be detected as early as $2-4 \mathrm{~h}$ after kidney injury $[19,20]$. Therefore, the present study used two measurements of serum NGAL one taken immediately after ROSC (ROSC-NGAL) and another reading at $3 \mathrm{~h}$ after ROSC ( $3 \mathrm{~h}-\mathrm{NGAL})$ - to determine the usefulness of NGAL as an early predictor of AKI.

Data on baseline patient characteristics, including sex, age, and medical history, were collected. The following factors were identified with regard to the cardiac arrest event: initial rhythm, witnessed cardiac arrest, bystander cardiopulmonary resuscitation (CPR), time from emergency medical service (EMS) activation to arrival of EMS, time from EMS activation to first defibrillation, time to ROSC, and dose of epinephrine used during CPR.

To determine the post-ROSC patient condition, we collected information on the following factors: continuous renal replacement therapy, coronary angiography, duration of TTM and targeted body temperature, survival at discharge, CPC at discharge, and 1- and 6month survival.

\section{Statistical analysis}

Data are expressed as median with interquartile ranges for continuous data with non-normal distribution, and as the number with percentages for categorical variables. The study population was divided into two subgroups based on AKI occurrence and neurologic outcome at discharge, and intergroup comparisons of general characteristics and clinical findings were undertaken. For items that required statistical verification, the Mann-Whitney $U$ test was used for continuous variables, and the chi-square or Fisher's exact test was used for categorical variables. Binary logistic regression analysis was used to assess the predictor variables that were identified on univariate analyses. Odds ratios (ORs) and 95\% confidence intervals (CIs) were computed from the estimated coefficients in the regression model. All statistical analyses were conducted in SPSS version 21.0 for Windows (SPSS Inc., Chicago, IL, USA). The adjusted ORs and 95\% CIs were obtained from multivariate analyses. Furthermore, to determine the predictive performance of a significant variable for AKI, a receiver operating characteristic (ROC) curve analysis was created by using MedCalc Statistical Software version 19 (MedCalc Software BVBA, Ostend, Belgium). Moreover, the areas under the ROC curve (AUROCs) and 95\% CIs were calculated, and the AUROCs were compared by DeLong's method [21]. A two-tailed $p$-value of $<0.05$ was considered statistically significant.

\section{Results}

During the study period, a total of 861 OHCA patients were admitted to the emergency room, and 97 of them received TTM. After excluding patients without NGAL measurement values and patients with chronic kidney disease, 89 patients (mean age 53.8 years; 63 males [70.8\%]) were included in the final study population (Fig. 1).

\section{General characteristics of study participants}

The general characteristics (Table 1) were compared between the two study groups, which were stratified by the presence or absence of AKI (AKI (+) group vs AKI (-) group). The AKI (+) group included 48 patients (55.1\%). The AKI (-) group included 40 patients (44.9\%). There were no significant intergroup differences in the sex distribution, mean age, and presence of underlying diseases. With regard to the initial rhythm, the AKI (+) group showed a significantly higher asystole rate (51.1\%), whereas the AKI (-) group showed a higher incidence of ventricular fibrillation (Vf; 66.7\%) and pulseless electrical activity (PEA; 23.1\%). The rate of witnessed cardiac arrest was higher in the AKI (-) than in the AKI (+) group $(92.5 \%$ vs $59.2 \%)$, whereas significantly higher epinephrine doses were used in the AKI (+) group during CPR. Furthermore, there were no significant intergroup differences with regard to bystander CPR, time from EMS activation to EMS arrival, time from EMS activation to first defibrillation, and time until ROSC. The general characteristics were analysed according to the AKI stage 


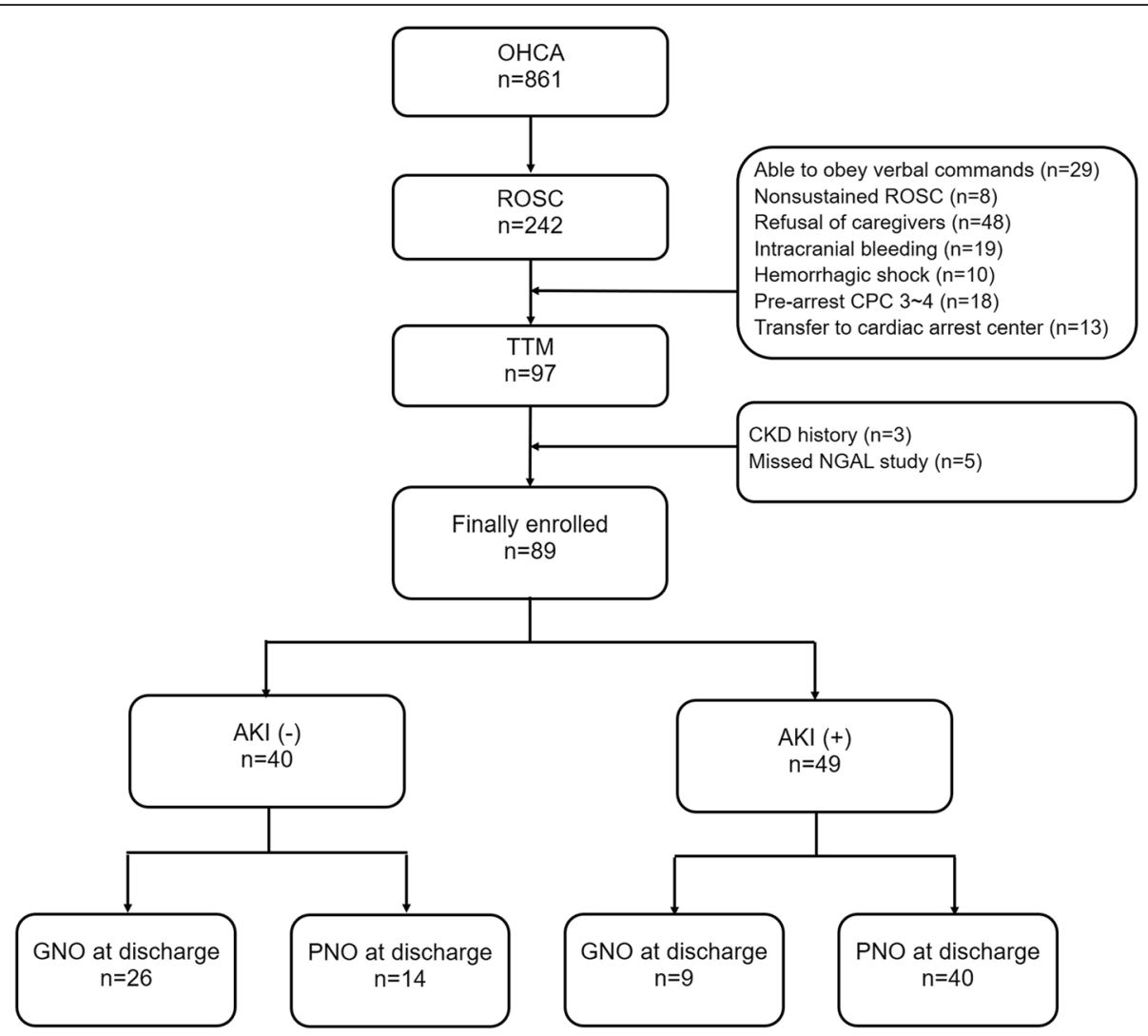

Fig. 1 Flow chart of the study population. OHCA out-of-hospital cardiac arrest: ROSC return of spontaneous circulation: TTM targeted temperature management: CKD chronic kidney disease: NGAL neutrophil gelatinase-associated lipocalin: AKI acute kidney injury: GNO good neurologic outcome: PNO poor neurologic outcome

(Additional file 1). In the AKI (+) group, the proportion of stages 1, 2, and 3 AKI was 23.6, 13.5, and 18.0\% respectively. Compared to the AKI (+) vs AKI (-) group analysis, a similar trend was seen in the AKI stage analysis except for the medical history of diabetes mellitus. With regard to diabetes mellitus, there was no significant difference in the distribution of the incidence in the AKI (+) and AKI (-) groups ( $p$-value 0.202), although there was a significant difference in the distribution of groups by the AKI stages $(p=0.025$, AKI $(-)$ group, 12.5\%; AKI Stage 1, 9.5\%; AKI Stage 2, 41.7\%: and AKI Stage 3, 37.5\%). There was no significant different in baseline creatinine values among the groups stratified by the AKI stage. The peak creatinine level was significantly different according to the AKI stages: 1.35 (95\% CI 1.04-1.26) for Stage 1, 2.11 (95\% CI 1.99-2.38) for Stage 2, and 4.66 (95\% CI 3.70-7.38) for Stage 3.

The intergroup differences in neurologic outcomes at discharge (GNO group vs PNO group) showed that the PNO group (CPC 3-5) included 54 patients (60.7\%). There were no significant between-group differences in the sex distribution, mean age, and presence of underlying diseases in the GNO and PNO groups. Similar to the AKI (+) group, the PNO group showed a significantly higher asystole rate (48.1\%), whereas the GNO group showed higher rates of Vf (26.9\%) and PEA (25.0\%). The frequency of witnessed cardiac arrest was higher in the GNO group (91.4\% vs 63.0\%), whereas time to ROSC was longer in the PNO group (30.5 [14.0-37.3] min vs 16.5 [10.5-29.5] $\mathrm{min}$ ). The epinephrine dose used in CPR was significantly higher in the PNO group. However, there were no significant differences in bystander CPR, time from EMS activation to EMS arrival, and time from EMS activation to first defibrillation.

\section{Comparison of clinical characteristics according to AKI occurrence and neurological outcome}

The clinical characteristics were compared according to AKI occurrence and neurological outcome (Table 2). The ROSC-NGAL and $3 \mathrm{~h}$-NGAL were significantly higher in the AKI (+) group. In addition, the AKI (-) group showed a significantly higher percentage of survival at discharge $(85.0 \%$ vs $28.6 \%)$. The percentage of poor outcome based on the CPC score was higher in the 
Table 1 General characteristics of study patients

\begin{tabular}{|c|c|c|c|c|c|c|c|c|c|c|c|c|}
\hline \multirow{3}{*}{$\begin{array}{l}\text { Characteristics } \\
\text { Number of patients }\end{array}$} & \multirow{2}{*}{\multicolumn{2}{|c|}{ Total }} & \multicolumn{5}{|c|}{ Acute kidney injury } & \multicolumn{5}{|c|}{ Neurologic outcome at discharge } \\
\hline & & & \multicolumn{2}{|l|}{$\overline{(-)}$} & \multicolumn{2}{|l|}{$(+)$} & \multirow[t]{2}{*}{$p$-value } & \multicolumn{2}{|c|}{ Good } & \multicolumn{2}{|l|}{ Poor } & \multirow[t]{2}{*}{$p$-value } \\
\hline & 89 & $(100.0)$ & 40 & $(44.9)$ & 49 & $(55.1)$ & & 35 & $(39.3)$ & 54 & $(60.7)$ & \\
\hline Sex & & & & & & & 0.430 & & & & & 0.124 \\
\hline Male & 63 & $(70.8)$ & 30 & $(75.0)$ & 33 & $(67.3)$ & & 28 & $(80.0)$ & 35 & $(64.8)$ & \\
\hline Female & 26 & $(29.2)$ & 10 & $(25.0)$ & 16 & $(32.7)$ & & 7 & $(20.0)$ & 19 & $(35.2)$ & \\
\hline Age (years) & 56.0 & $(43.5-66.0)$ & 53.0 & $(43.0-62.0)$ & 58.0 & $(44.5-67.0)$ & 0.358 & 55.0 & $(43.0-62.0)$ & 56.0 & $(44.8-68.0)$ & 0.245 \\
\hline \multicolumn{13}{|l|}{ Medical history } \\
\hline HTN & 28 & $(31.5)$ & 11 & $(27.5)$ & 17 & $(34.7)$ & 0.467 & 13 & $(37.1)$ & 15 & $(27.8)$ & 0.353 \\
\hline DM & 18 & $(20.2)$ & 5 & $(12.5)$ & 13 & $(26.5)$ & 0.101 & 7 & $(20.0)$ & 11 & $(20.4)$ & 0.966 \\
\hline $\mathrm{HF}$ & 2 & $(2.2)$ & 1 & $(2.5)$ & 1 & $(2.0)$ & 1.000 & 1 & $(2.9)$ & 1 & $(1.9)$ & 1.000 \\
\hline Initial rhythm by EMS or hospital & & & & & & & $<0.001$ & & & & & $<0.001$ \\
\hline Vf & 40 & $(44.9)$ & 26 & $(65.0)$ & 14 & $(28.6)$ & & 26 & $(74.3)$ & 14 & $(25.9)$ & \\
\hline PEA & 18 & $(20.2)$ & 9 & $(22.5)$ & 9 & $(18.4)$ & & 5 & $(14.3)$ & 13 & $(24.1)$ & \\
\hline Asystol & 28 & $(31.5)$ & 4 & $(10.0)$ & 24 & $(49.0)$ & & 3 & $(8.6)$ & 25 & $(46.3)$ & \\
\hline Unknown & 3 & $(3.4)$ & 1 & $(2.5)$ & 2 & $(4.1)$ & & 1 & $(2.9)$ & 2 & $(3.7)$ & \\
\hline Witness cardiac arrest & 66 & $(74.2)$ & 37 & $(92.5)$ & 29 & $(59.2)$ & $<0.001$ & 32 & $(91.4)$ & 34 & $(63.0)$ & 0.003 \\
\hline Bystander CPR & 49 & $(55.1)$ & 25 & $(62.5)$ & 24 & $(49.0)$ & 0.202 & 20 & $(57.1)$ & 29 & $(53.7)$ & 0.750 \\
\hline EMS activation to EMS arrival (min) & 7.0 & $(5.6-10.0)$ & 7.0 & $(6.0-9.0)$ & 7.0 & $(5.0-10.0)$ & 0.922 & 6.0 & $(5.0-10.0)$ & 8.0 & $(6.0-10.0)$ & 0.305 \\
\hline $\begin{array}{l}\text { EMS activation to first defibrillation } \\
\text { (min) }\end{array}$ & 7.5 & $(6.0-12.0)$ & 7.5 & $(6.0-10.0)$ & 7.5 & $(5.6-12.3)$ & 1.000 & 7.0 & $(6.0-10.0)$ & 9.0 & $(6.0-12.0)$ & 0.389 \\
\hline Time to ROSC (min) & 25.0 & $(13.8-35.0)$ & 19.0 & $(11.5-33.5)$ & 27.0 & $(14.0-36.5)$ & 0.410 & 16.5 & $(10.5-29.5)$ & 30.5 & $(14.8-37.3)$ & 0.007 \\
\hline Epinephrine dose during CPR & 1.0 & $(0.0-3.0)$ & 0.0 & $(0.0-1.0)$ & 3.0 & $(1.0-4.0)$ & $<0.001$ & 0.0 & $(0.0-0.0)$ & 2.5 & $(1.0-4.0)$ & $<0.001$ \\
\hline
\end{tabular}

Quantitative data are expressed as median (interquartile range), categorical data are presented as number of subjects (percentages). Mann-Whitney $U$ test was used for continuous variable analysis, while chi-squared test or Fisher's exact test were used for categorical variable analysis as appropriate $H T N$ hypertension, DM diabetes mellitus, HF heart failure, Vf ventricular fibrillation, PEA pulseless electrical activity, CPR cardiopulmonary resuscitation, EMS emergency medical system, ROSC return of spontaneous circulation

AKI (+) group (81.6\% vs $35.0 \%)$. Furthermore, the rates of 1- and 6-month survival were significantly higher in the AKI (-) group (1-month survival: $82.5 \%$ vs $28.6 \%$; 6 month survival: $82.5 \%$ vs 29.2\%; Table 2 and Fig. 2). Furthermore, the clinical characteristics according to the AKI stage were analysed (Additional file 1). Compared to the AKI (+) vs AKI (-) group analysis, a similar trend was observed in the sub-analysis by the AKI stage.

Intergroup comparison of the ROSC-NGAL and $3 \mathrm{~h}$ NGAL levels in the groups stratified by the neurologic outcome at discharge showed significantly higher levels of NGAL in the PNO group. The percentage of patients who needed continuous renal replacement therapy was significantly higher in the PNO group $(23.1 \%$ vs $3.6 \%)$, whereas a significantly higher percentage of patients in the GNO group underwent coronary angiography (85.7\% vs 18.5\%; Table 2 and Fig. 2).

\section{Univariate and multivariate logistic regression analysis for AKI}

To examine the predictors of AKI, we undertook logistic regression analysis to identify the factors that significantly differed between the AKI (+) and AKI (-) groups. The results of multivariate logistic regression analysis showed that unwitnessed cardiac arrest (OR 8.274; 95\% CI 1.287-53.18) and 3 h-NGAL (OR 1.022; 95\% CI 1.009-1.035) were significantly associated with AKI (Table 3).

To examine the predictors of poor neurologic outcome at discharge, we conducted a logistic regression analysis to identify factors that showed significant differences between the good and poor outcome groups. Multivariate logistic regression analysis showed that unwitnessed cardiac arrest (OR 8.357; 95\% CI 1.211-57.654) and the higher dose of epinephrine used during CPR (OR 3.348; 95\% CI 1.465-7.652) were significantly associated with poor neurologic outcome (Table 3).

\section{ROC curve and cut-off value of NGAL at 3 hours after ROSC for AKI}

A ROC curve analysis was conducted to verify the clinical usefulness of $3 \mathrm{~h}-\mathrm{NGAL}$ as a predictor of AKI. The AUROC of $3 \mathrm{~h}$-NGAL for AKI was 0.910 (95\% CI 
Table 2 Clinical characteristics after return of spontaneous circulation

\begin{tabular}{|c|c|c|c|c|c|c|c|c|c|c|c|c|}
\hline & Total & & Acute & kidney injur & & & & Neuro & ologic outcon & me at d & lischarge & \\
\hline & & & $(-)$ & & $(+)$ & & $p$-value & Good & & Poor & & $p$-value \\
\hline NGAL at ROSC & 124.0 & $(96.0-186.0)$ & 105.5 & $(83.3-143.3)$ & 142.0 & $(107.0-263.0)$ & 0.001 & 105.0 & $(84.0-141.0)$ & 140.0 & $(140.6-245.8)$ & 0.002 \\
\hline NGAL at $3 \mathrm{~h}$ after ROSC & 181.0 & $(115.0-381.0)$ & 115.0 & $(84.3-145.5)$ & 353.0 & $(223.0-510.0)$ & $<0.001$ & 134.0 & $(84.0-181.0)$ & 255.5 & $(139.5-434.8)$ & $<0.001$ \\
\hline CRRT & 11 & $(14.7)$ & 0 & $(0.0)$ & 11 & $(23.4)$ & 0.005 & 1 & (3.6) & 10 & $(23.1)$ & 0.045 \\
\hline CAG & 40 & $(44.9)$ & 25 & $(62.5)$ & 15 & $(30.6)$ & 0.003 & 30 & $(85.7)$ & 10 & $(18.5)$ & $<0.001$ \\
\hline Target temperature & & & & & & & 0.586 & & & & & 0.559 \\
\hline $33^{\circ} \mathrm{C}$ & 86 & (96.6) & 38 & $(95.0)$ & 48 & $(98.0)$ & & 33 & $(94.3)$ & 53 & $(98.1)$ & \\
\hline$<36^{\circ} \mathrm{C}$ & 3 & (3.4) & 2 & $(5.0)$ & 1 & $(2.0)$ & & 2 & $(5.7)$ & 1 & (1.9) & \\
\hline TTM duration & & & & & & & 0.624 & & & & & 1.000 \\
\hline $24 \mathrm{~h}$ & 85 & $(95.5)$ & 39 & $(97.5)$ & 46 & $(93.9)$ & & 34 & $(97.1)$ & 51 & $(94.4)$ & \\
\hline $48 \mathrm{~h}$ & 4 & $(4.5)$ & 1 & $(2.5)$ & 3 & $(6.1)$ & & 1 & $(2.9)$ & 3 & $(5.6)$ & \\
\hline Survival discharge & 48 & $(53.9)$ & 34 & $(85.0)$ & 14 & (28.6) & $<0.001$ & 35 & $(100.0)$ & 13 & $(24.1)$ & $<0.001$ \\
\hline CPC at discharge & & & & & & & $<0.001$ & & & & & \\
\hline Good $(1,2)$ & 35 & (39.3) & 26 & $(65.0)$ & 9 & $(18.4)$ & & & & & & \\
\hline Poor $(3,4,5)$ & 54 & $(60.7)$ & 14 & $(35.0)$ & 40 & (81.6) & & & & & & \\
\hline Survival at 1 month & 47 & $(52.8)$ & 33 & $(82.5)$ & 14 & $(28.6)$ & $<0.001$ & 35 & $(100.0)$ & 12 & $(22.2)$ & $<0.001$ \\
\hline Survival at 6 months & 47 & $(53.4)$ & 33 & $(82.5)$ & 14 & $(29.2)$ & $<0.001$ & 35 & $(100.0)$ & 12 & $(22.6)$ & $<0.001$ \\
\hline
\end{tabular}

Quantitative data are expressed as median (interquartile range), categorical data are presented as number of subjects (percentages). Mann-Whitney $U$ test was used for continuous variable analysis, while chi-squared test or Fisher's exact test were used for categorical variable analysis as appropriate

NGAL neutrophil gelatinase-associated lipocalin, ROSC return of spontaneous circulation, CRRT continuous renal replacement therapy, CAG coronary angiography,

TTM targeted temperature management, CPC cerebral performance category

0.830-0.960), whereas the cut-off value was $178 \mathrm{ng} / \mathrm{mL}$. Therefore, the sensitivity, specificity, positive likelihood ratio, and negative likelihood ratio for AKI were 83.67 (95\% CI 70.3-92.7), 90.00 (95\% CI 76.3-97.2), 8.37 (95\% CI 3.3-21.4), and 0.18 (95\% CI $0.10-0.3$ ), respectively. The AUROC of ROSC-NGAL was 0.698 (95\% CI 0.5910.791 ), which was significantly lower than that of $3 \mathrm{~h}$ NGAL ( $p$-value = 0.0001; Fig. 3).

\section{Discussion}

This study aimed to compare and analyse the NGAL levels at ROSC and $3 \mathrm{~h}$ post ROSC in AKI among patients who underwent TTM, and to evaluate the clinical utility of NGAL as a predictive marker for AKI. Moreover, the study aimed to verify the association between neurologic outcome and the ROSC-NGAL and $3 \mathrm{~h}$ NGAL levels.
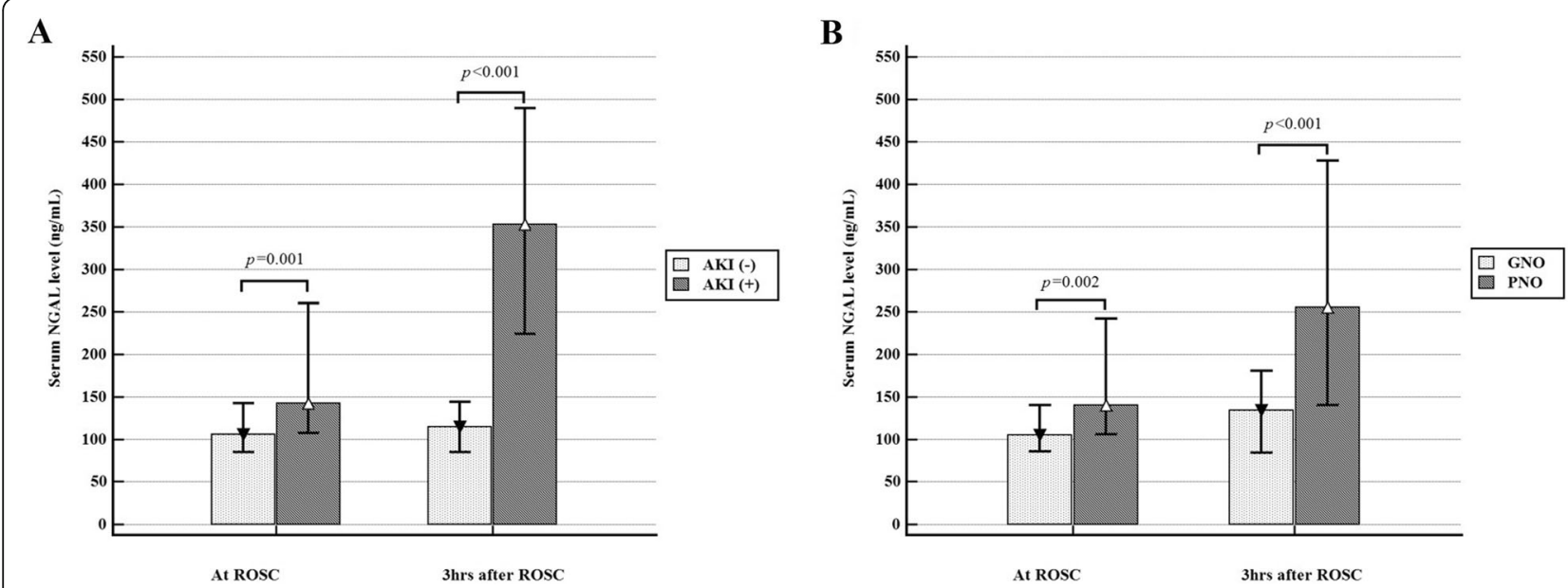

Fig. 2 a. Serum NGAL level at immediately and $3 \mathrm{~h}$ after ROSC according to acute kidney injury development. b. Serum NGAL level at immediately and $3 \mathrm{~h}$ after ROSC according to neurologic outcome at discharge. NGAL neutrophil gelatinase-associated lipocalin: ROSC return of spontaneous circulation: AKI acute kidney injury GNO good neurologic outcome: PNO poor neurologic outcome 
Table 3 Univariable and multivariable logistic regression analysis for acute kidney injury and poor neurologic outcome at discharge

\begin{tabular}{|c|c|c|c|c|c|c|}
\hline \multirow[t]{2}{*}{ Variables } & \multicolumn{3}{|c|}{ Univariable } & \multicolumn{3}{|c|}{ Multivariable } \\
\hline & $\overline{\mathrm{OR}}$ & $(95 \% \mathrm{Cl})$ & $\overline{p \text {-value }}$ & $\overline{\mathrm{OR}}$ & $(95 \% \mathrm{Cl})$ & $p$-value \\
\hline \multicolumn{7}{|l|}{ Acute kidney injury } \\
\hline Non-shockable rhythm & 4.714 & $(1.891-11.750)$ & 0.001 & 2.963 & $(0.546-16.090)$ & 0.208 \\
\hline Witness cardiac arrest: No & 8.506 & $(2.301-31.437)$ & 0.001 & 8.274 & $(1.287-53.18)$ & 0.026 \\
\hline Epinephrine dose during CPR & 1.922 & $(1.362-2.713)$ & $<0.001$ & 1.282 & $(0.830-1.979)$ & 0.263 \\
\hline NGAL at ROSC & 1.009 & $(1.002-1.017)$ & 0.014 & 0.988 & $(0.969-1.008)$ & 0.230 \\
\hline NGAL at $3 \mathrm{~h}$ after ROSC & 1.019 & $(1.011-1.028)$ & $<0.001$ & 1.022 & $(1.009-1.035)$ & 0.001 \\
\hline \multicolumn{7}{|c|}{ Poor neurologic outcome at discharge } \\
\hline Non-shockable rhythm & 8.821 & $(3.240-24.020)$ & $<0.001$ & 3.005 & $(0.627-14.396)$ & 0.169 \\
\hline Witness cardiac arrest: No & 6.275 & $(1.700-23.161)$ & 0.006 & 8.357 & $(1.211-57.654)$ & 0.031 \\
\hline Time to ROSC & 1.057 & $(1.014-1.102)$ & 0.008 & 1.021 & $(0.962-1.084)$ & 0.495 \\
\hline Epinephrine dose during CPR & 3.833 & $(2.056-7.146)$ & $<0.001$ & 3.348 & $(1.465-7.652)$ & 0.004 \\
\hline NGAL at ROSC & 1.011 & $(1.002-1.019)$ & 0.014 & 1.017 & $(0.998-1.036)$ & 0.084 \\
\hline NGAL at $3 \mathrm{~h}$ after ROSC & 1.004 & $(1.001-1.007)$ & 0.022 & 0.997 & $(0.992-1.001)$ & 0.113 \\
\hline
\end{tabular}

CPR cardiopulmonary resuscitation, NGAL neutrophil gelatinase-associated lipocalin, ROSC return of spontaneous circulation

AKI is associated with poor clinical outcome in PCAS patients, and the incidence of AKI in PCAS patients ranges from 12 to $80 \%$ [3-7]. In a study by Oh et al. [3] that was published in 2019, AKI occurred in 348 (60\%) of 583 patients who underwent TTM and was associated with poor neurologic outcome at 6 months (adjusted OR 0.206; 95\% CI 0.099-0.426; $p<0.001$ ). In a 2015 study by Geri et al. [4], Stage 3 AKI occurred in 280 out of 580 OHCA patients (48.3\%) and was associated with the 30-day mortality rate (OR 1.60; 95\% CI 1.05-2.43; $p=$ 0.03). A similar tendency was identified in this study,

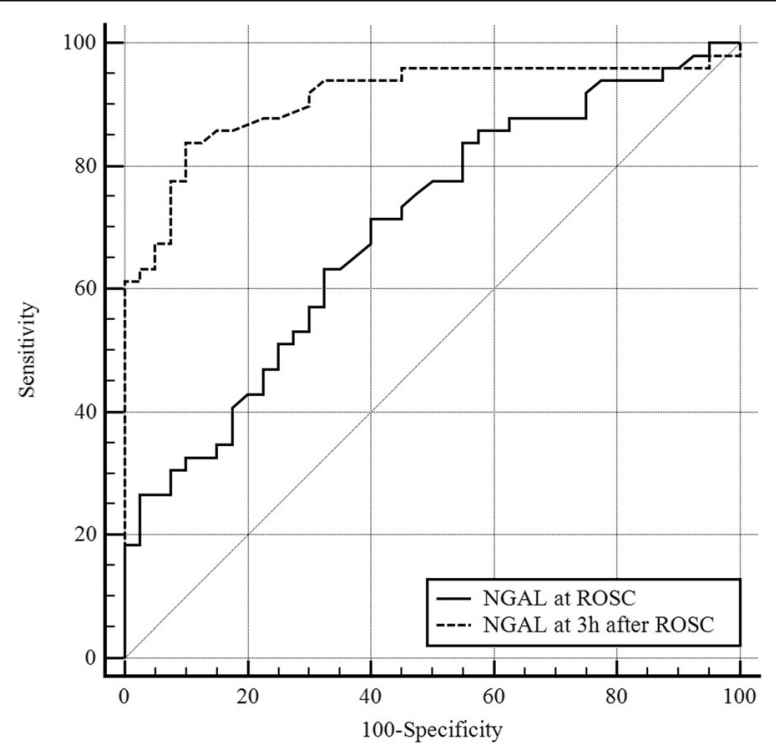

Fig. 3 ROC curve of NGAL for acute kidney injury development. NGAL neutrophil gelatinase-associated lipocalin: ROSC return of spontaneous circulation wherein a comparison of survival at discharge, CPC at discharge, and the 1- and 6-month survival in the AKI $(+)$ and AKI (-) groups showed a significantly higher frequency of poor outcome in the AKI (+) group.

The appropriate management of AKI, which has an effect on poor prognosis in PCAS patients, requires the prediction and early management of AKI. In the results reported from a study by Choi et al. [22] in 2020, AKI occurred in $55.5 \%$ of 1373 patients who underwent TTM after OHCA, and 78.1\% of those patients developed AKI within 3 days after OHCA. Because there is no specific treatment to reverse AKI, early recognition and management are important to improve the clinical outcomes. The recognition of patients at risk for AKI, or those with possible AKI but before the appearance of clinical manifestations, is likely to result in better outcomes than that attained by treating only patients with established AKI [17]. The management of patients at risk for AKI includes the discontinuation of all nephrotoxic agents when possible, ensuring volume status and perfusion pressure, considering functional hemodynamic monitoring, monitoring $\mathrm{SCr}$ and urine output, avoiding hyperglycaemia, and considering alternatives to radiocontrast procedures [17]. Moreover, early recognition of AKI is a useful indicator to secure the requisite medical resources, such as CRRT. In the clinical practice guidelines for AKI in 2012, the KDIGO Acute Kidney Injury Work Group emphasised the importance of studies on biomarkers for the early diagnosis, prognosis, and differential diagnosis of AKI [17]. Thus, additional studies are needed on biomarkers for the early diagnosis or risk prediction of AKI, as well as the prediction of mortality or long-term renal replacement therapy in AKI patients. The factors that are being studied as biomarkers for AKI 
include NGAL, cystatin C, interleukin-18, kidney injury molecule-1, and plasma IL-6. Among the biomarkers of AKI, both NGAL and gamma-glutamyl transpeptidase/ alkaline phosphatase have evidence from Phase 4 or higher studies [8].

Human NGAL was originally identified as a novel protein that was isolated from the secondary granules of human neutrophils [23]. Preclinical transcriptome profiling in a number of AKI models revealed NGAL to be one of the most robustly upregulated genes in the kidney post injury $[24,25]$. The NGAL has been identified as a useful marker for the early prediction of AKI in situations that confer a potential risk of kidney injury, such as cardiopulmonary bypass, contrast administration, and kidney transplantation [19]. Cardiac surgery-associated AKI is indicated by a more than 10-fold elevation in the urinary and serum levels of NGAL within 2-6 h after surgery. Many prospective studies have reported that patients with AKI showed significantly increased NGAL levels at 1-3 h after surgery [26-29]. Contrast-induced AKI could be predicted by NGAL measurement at $2 \mathrm{~h}$ after contrast administration [20, 30-32]. In studies that analysed the association of NGAL in patients with post-OHCA AKI, the timepoint of NGAL varied from immediately after ROSC to several days later, and there were differences in AUROCs or ORs of NGAL for AKI depending on the time of measurement [13-15]. However, no studies have comparatively evaluated NGAL values that were measured immediately after ROSC with those measured hours later. This study focused on the early prediction of AKI, and compared ROSC-NGAL and $3 \mathrm{~h}-\mathrm{NGAL}$ to determine the clinical utility of NGAL as a predictor of AKI. The results showed that $3 \mathrm{~h}-\mathrm{NGAL}$ is a more accurate predictor of AKI than ROSC-NGAL. Meanwhile, a study has been published recently which refuted prevailing opinion that NGAL is produced in the kidneys. A study by Skrypnyk et al. showed that an increase in IL-6 in wild-type mice with ischemic AKI induces the hepatic production of NGAL, thereby increasing the plasma NGAL levels. Based on their result, they predicted that NGAL levels may increase regardless of whether AKI occurs in conditions wherein plasma IL-6 levels can increase. Those authors reported the need for further studies to validate this result with regard to AKI in the general [12].

Research has been actively undertaken on NGAL and clinical outcomes in OHCA patients. In a 2019 study by Lee et al. [33], the plasma NGAL measured $4 \mathrm{~h}$ after ROSC among adult OHCA patients who were treated with TTM was associated with both the neurologic outcome at the time of discharge (adjusted OR 1.004; 95\% CI $1.001-1.007$ ) as well as the 28-day mortality rate (adjusted OR 1.003; 95\% CI 1.001-1.004). In 2018, Park et al. [34] reported that the NGAL level was measured immediately and 24,46 , and $72 \mathrm{~h}$ after ROSC and was analysed to predict the long-term outcome and survival in 76 OHCA patients who underwent TTM; the results showed that the NGAL value measured after $72 \mathrm{~h}$ was the optimal predictive indicator for the outcome and survival $(\mathrm{AUROC}=$ $0.72 ; p=0.02$ ). In a 2017 study, Kaneko et al. [35] analysed the neurologic outcome at discharge based on NGAL measurements at 1 and 2 days after ROSC; the NGAL level after 2 days showed a comparable predictive value as the 2-day neuron-specific enolase, which has widespread application in the prediction of the neurologic outcome. In this study, the results of multivariate regression analysis of ROSC-NGAL and $3 \mathrm{~h}$-NGAL measurements did not identify them as significant risk factors for poor neurologic outcome (ROSC-NGAL: OR 1.017; 95\% CI 0.998-1.036; $p=0.084$, 3 h-NGAL: OR 0.997; 95\% CI 0.992-1.001; $p=$ $0.113)$. The neuroprognostic value of NGAL measured within $24 \mathrm{~h}$ is remains controversial [34]; therefore, additional studies are necessary to determine the optimal timepoints for NGAL measurement after ROSC.

This study had several limitations. First, in patients with missing data for the serum creatinine level, we used the creatinine level on the first day of hospitalisation as the baseline value. Second, the study did not consider the potential effect of the history of concurrent medications and the radiocontrast procedure on renal function, volume status after admission, and the onset of complications. Third, the single-centre study design is another limitation of this study. Forth, number of study patients was low. The frequency of OHCA is extremely low, and this limitation is difficult to avoid due to the nature of the disease. However, this drawback may be circumvented by multicentre research studies. Fourth, a recent study reported the hepatic production of NGAL via the IL-6 level. Therefore, the results of this study should be interpreted with due consideration to conditions that may increase IL- 6 as well as the risk of AKI.

\section{Conclusions}

The serum NGAL concentration measured at $3 \mathrm{~h}$ after ROSC is an excellent early predictive marker for AKI in OHCA patients treated with TTM. Further research is needed to identify the optimal timepoint of measurement to establish NGAL as a predictor of the neurologic outcome and to validate the findings of this research.

\section{Supplementary information}

Supplementary information accompanies this paper at https://doi.org/10. 1186/s12882-020-02054-7.

Additional file 1. General and clinical characteristics of study patients according to AKI stages

\section{Abbreviations}

NGAL: Neutrophil gelatinase-associated lipocalin; AKI: Acute kidney injury; ROSC: Return of spontaneous circulation; OHCA: Out-of-hospital cardiac arrest; TTM: Targeted temperature management; PCAS: Post-cardiac arrest 
syndrome; IL-6: Interleukin-6; KDIGO: Kidney Disease Improving Global Outcomes; SCr: Serum creatinine; CPC: Cerebral performance category; GNO: Good neurologic outcome; PNO: Poor neurologic outcome; CPR: Cardiopulmonary resuscitation; EMS: Emergency medical service; OR: Odds ratio; Cl: Confidence interval; ROC: Receiver operating characteristics; AUROC: Area under the receiver operating characteristics curve; Vf: Ventricular fibrillation; PEA: Pulseless electrical activity

\section{Acknowledgments}

None.

\section{Authors' contributions}

Conception and design: DHL and YHC. Acquisition: analysis: and interpretation of data: YHC and JHL. Drafting the manuscript for intellectual content: JHL and YHC. Statistical analysis: JHL. All authors reviewed: revised: and approved the manuscript for submissions. Study supervision: DHL and YHC. The authors read and approved the final manuscript.

\section{Funding}

The authors received no financial support for the research: authorship: and/ or publication of this article.

\section{Availability of data and materials}

The datasets used and/or analysed during the current study are available from the corresponding author on reasonable request.

\section{Ethics approval and consent to participate}

This study was approved by the institutional review board of Ewha Womans University Mokdong Hospital. In the case of TTM patients, the patient's own consent cannot be obtained. Written informed consent was obtained from legal guardians of the patients before enrollment in the study.

\section{Consent for publication}

Not applicable.

\section{Competing interests}

The authors declare that they have no competing interests.

\section{Author details}

'Department of Emergency Medicine, College of Medicine, Ewha Womans University Mokdong Hospital, 1071 Anyangcheon-ro, Yangcheon-gu, Seoul 07985, South Korea. ${ }^{2}$ Department of Emergency Medicine, Chung-Ang University, College of Medicine, Seoul, South Korea.

Received: 11 June 2020 Accepted: 2 September 2020

Published online: 07 September 2020

\section{References}

1. Stub D, Bernard S, Duffy SJ, Kaye DM. Post cardiac arrest syndrome: a review of therapeutic strategies. Circulation. 2011;123(13):1428-35.

2. Nolan JP, Soar J, Cariou A, Cronberg T, Moulaert VR, Deakin CD, Bottiger BW, Friberg H, Sunde K, Sandroni C. European Resuscitation Council and European Society of Intensive Care Medicine Guidelines for Postresuscitation Care 2015: Section 5 of the European resuscitation council guidelines for resuscitation 2015. Resuscitation. 2015(95):202-22.

3. Oh JH, Lee DH, Cho IS, Youn CS, Lee BK, Wee JH, Cha KC, Chae MK, Shin J. Korean hypothermia network I: association between acute kidney injury and neurological outcome or death at 6months in out-of-hospital cardiac arrest: a prospective, multicenter, observational cohort study. J Crit Care. 2019:54: 197-204.

4. Geri G, Guillemet L, Dumas F, Charpentier J, Antona M, Lemiale V, Bougouin W, Lamhaut L, Mira J-P, Vinsonneau C. Acute kidney injury after out-ofhospital cardiac arrest: risk factors and prognosis in a large cohort. Intensive Care Med. 2015;41(7):1273-80.

5. Beitland S, Nakstad E, Stær-Jensen H, Draegni T, Andersen G, Jacobsen D, Brunborg C, Waldum-Grevbo B, Sunde K. Impact of acute kidney injury on patient outcome in out-of-hospital cardiac arrest: a prospective observational study. Acta Anaesthesiol Scand. 2016;60(8):1170-81.

6. Sandroni C, Dell'Anna AM, Tujjar O, Geri G, Cariou A, Taccone FS. Acute kidney injury (AKI) after cardiac arrest: a systematic review and meta-analysis of clinical studies. Minerva Anestesiol. 2016;82(9):989-99.
7. Storm C, Krannich A, Schachtner T, Engels M, Schindler R, Kahl A, Otto NM Impact of acute kidney injury on neurological outcome and long-term survival after cardiac arrest - a 10 year observational follow up. J Crit Care. 2018:47:254-9.

8. Siew ED, Ware LB, Ikizler TA. Biological markers of acute kidney injury. J Am Soc Nephrol. 2011;22(5):810-20.

9. Soni SS, Cruz D, Bobek I, Chionh CY, Nalesso F, Lentini P, de Cal M, Corradi V, Virzi G, Ronco C. NGAL: a biomarker of acute kidney injury and other systemic conditions. Int Urol Nephrol. 2010;42(1):141-50.

10. Khawaja S, Jafri L, Siddiqui I, Hashmi M, Ghani F. The utility of neutrophil gelatinase-associated Lipocalin (NGAL) as a marker of acute kidney injury (AKI) in critically ill patients. Biomarker research. 2019;7(1):4.

11. Paragas N, Qiu A, Zhang Q, Samstein B, Deng S-X, Schmidt-Ott KM, Viltard M, Yu W, Forster CS, Gong G. The Ngal reporter mouse detects the response of the kidney to injury in real time. Nat Med. 2011;17(2):216.

12. Skrypnyk NI, Gist KM, Okamura K, Montford JR, You Z, Yang H, Moldovan R, Bodoni E, Blaine JT, Edelstein CL, et al. IL-6-mediated hepatocyte production is the primary source of plasma and urine neutrophil gelatinase-associated lipocalin during acute kidney injury. Kidney Int. 2020;97(5):966-79.

13. Park SO, Ahn JY, Lee YH, Kim YJ, Min YH, Ahn HC, Sohn YD, Park SM, Oh YT, Shin DH. Plasma neutrophil gelatinase-associated lipocalin as an early predicting biomarker of acute kidney injury and clinical outcomes after recovery of spontaneous circulation in out-of-hospital cardiac arrest patients. Resuscitation. 2016;101:84-90.

14. Cho YS, Lee BK, Lee DH, Jung YH, Lee SM, Park JS, Jeung KW. Association of plasma neutrophil gelatinase-associated lipocalin with acute kidney injury and clinical outcome in cardiac arrest survivors depends on the time of measurement. Biomarkers. 2018;23(5):487-94.

15. Lee DH, Lee BK, Cho YS, Jung YH, Lee SM, Park JS, Jeung KW. Plasma neutrophil Gelatinase-associated Lipocalin measured immediately after restoration of spontaneous circulation predicts acute kidney injury in cardiac arrest survivors who underwent therapeutic hypothermia. Ther Hypothermia Temp Manag. 2018;8(2):99-107.

16. Holzer M. Targeted temperature management for comatose survivors of cardiac arrest. N Engl J Med. 2010;363(13):1256-64.

17. Group KDIGOAKIW. KDIGO clinical practice guideline for acute kidney injury. Kidney Int Suppl. 2012;2(1):1-138.

18. Jennett $B$, Bond M. Assessment of outcome after severe brain damage. Lancet. 1975;1(7905):480-4.

19. Devarajan P. Neutrophil gelatinase-associated lipocalin: a promising biomarker for human acute kidney injury. Biomark Med. 2010;4(2):265-80.

20. Bachorzewska-Gajewska H, Malyszko J, Sitniewska E, Malyszko JS, Dobrzycki S. Neutrophil-gelatinase-associated lipocalin and renal function after percutaneous coronary interventions. Am J Nephrol. 2006:26(3):287-92.

21. DeLong ER, DeLong DM, Clarke-Pearson DL. Comparing the areas under two or more correlated receiver operating characteristic curves: a nonparametric approach. Biometrics. 1988:837-45.

22. Choi YH, Lee DH, Oh JH, Wee JH, Jang TC, Choi SP, Park KN. Korean hypothermia network I: renal replacement therapy is independently associated with a lower risk of death in patients with severe acute kidney injury treated with targeted temperature management after out-of-hospital cardiac arrest. Crit Care. 2020;24(1):115.

23. Xu SY, Carlson M, Engstrom A, Garcia R, Peterson CG, Venge P. Purification and characterization of a human neutrophil lipocalin (HNL) from the secondary granules of human neutrophils. Scand J Clin Lab Invest. 1994; 54(5):365-76.

24. Devarajan P, Mishra J, Supavekin S, Patterson LT, Steven Potter S. Gene expression in early ischemic renal injury: clues towards pathogenesis, biomarker discovery, and novel therapeutics. Mol Genet Metab. 2003;80(4): 365-76.

25. Yuen PS, Jo SK, Holly MK, Hu X, Star RA. Ischemic and nephrotoxic acute renal failure are distinguished by their broad transcriptomic responses. Physiol Genomics. 2006;25(3):375-86.

26. Tuladhar SM, Puntmann VO, Soni M, Punjabi PP, Bogle RG. Rapid detection of acute kidney injury by plasma and urinary neutrophil gelatinaseassociated lipocalin after cardiopulmonary bypass. J Cardiovasc Pharmacol. 2009;53(3):261-6.

27. Haase-Fielitz A, Bellomo R, Devarajan P, Story D, Matalanis G, Dragun D, Haase M. Novel and conventional serum biomarkers predicting acute kidney injury in adult cardiac surgery--a prospective cohort study. Crit Care Med. 2009;37(2):553-60. 
28. Haase M, Bellomo R, Devarajan P, Ma Q, Bennett MR, Mockel M, Matalanis G, Dragun D, Haase-Fielitz A. Novel biomarkers early predict the severity of acute kidney injury after cardiac surgery in adults. Ann Thorac Surg. 2009; 88(1):124-30.

29. Wagener G, Jan M, Kim M, Mori K, Barasch JM, Sladen RN, Lee HT. Association between increases in urinary neutrophil gelatinase-associated lipocalin and acute renal dysfunction after adult cardiac surgery. Anesthesiology. 2006;105(3):485-91.

30. Hirsch R, Dent C, Pfriem H, Allen J, Beekman RH 3rd, Ma Q, Dastrala S, Bennett M, Mitsnefes M, Devarajan P. NGAL is an early predictive biomarker of contrast-induced nephropathy in children. Pediatr Nephrol. 2007;22(12): 2089-95.

31. Ling W, Zhaohui N, Ben H, Leyi G, Jianping L, Huili D, Jiaqi Q. Urinary IL-18 and NGAL as early predictive biomarkers in contrast-induced nephropathy after coronary angiography. Nephron Clin Pract. 2008:108(3):c176-81.

32. Bachorzewska-Gajewska H, Malyszko J, Sitniewska E, Malyszko JS, Dobrzycki S. Neutrophil gelatinase-associated lipocalin (NGAL) correlations with cystatin C, serum creatinine and eGFR in patients with normal serum creatinine undergoing coronary angiography. Nephrol Dial Transplant. 2007; 22(1):295-6.

33. Lee JH, Park I, You JS, Kim MJ, Lee HS, Park YS, Park HC, Chung SP Predictive performance of plasma neutrophil gelatinase-associated lipocalin for neurologic outcomes in out-of-hospital cardiac arrest patients treated with targeted temperature management: a prospective observational study. Medicine (Baltimore). 2019;98(34):e16930.

34. Park YR, Oh JS, Jeong H, Park J, Oh YM, Choi S, Choi KH. Predicting longterm outcomes after cardiac arrest by using serum neutrophil gelatinaseassociated lipocalin. Am J Emerg Med. 2018;36(4):660-4.

35. Kaneko T, Fujita M, Ogino Y, Yamamoto T, Tsuruta R, Kasaoka S. Serum neutrophil gelatinase-associated lipocalin levels predict the neurological outcomes of out-of-hospital cardiac arrest victims. BMC Cardiovasc Disord. 2017;17(1):111

\section{Publisher's Note}

Springer Nature remains neutral with regard to jurisdictional claims in published maps and institutional affiliations.

Ready to submit your research? Choose BMC and benefit from:

- fast, convenient online submission

- thorough peer review by experienced researchers in your field

- rapid publication on acceptance

- support for research data, including large and complex data types

- gold Open Access which fosters wider collaboration and increased citations

- maximum visibility for your research: over $100 \mathrm{M}$ website views per year

At $\mathrm{BMC}$, research is always in progress.

Learn more biomedcentral.com/submissions 